\title{
MODELING OCCUPANT ENERGY USE INTERVENTIONS IN EVOLVING SOCIAL NETWORKS
}

\author{
Kyle Anderson \\ SangHyun Lee \\ Civil and Environmental Engineering \\ University of Michigan \\ 2350 Hayward Street, G.G. Brown Building \\ Ann Arbor, MI 48109, USA
}

\begin{abstract}
Occupant behavior in buildings can contribute significantly to building energy demand and consumption. As a result, occupant behavior interventions to promote sustainability are becoming more widespread. Due to the expense in applying interventions, researchers have begun using computer simulations to analyze potential outcomes and better understand how complex systems can affect intervention success, in particular the effect of social network structure. In previous literature, studies have only evaluated social network effects using static social networks which are far from reality. Therefore, in this study we evaluate how a behavior intervention, here a comparative feedback system, is affected as social networks evolve over time using agent-based modeling. Results indicate that static social networks are much less volatile in their behavior and tend to have more convergent behavior relative to dynamic social networks. This implies that for normative interventions, dynamic networks have increased uncertainty in intervention outcome compared to static networks.
\end{abstract}

\section{INTRODUCTION}

It is predicted that continued increases in atmospheric concentrations of carbon dioxide caused by anthropogenic emissions will lead to significant changes in climate (Houghton et al. 2001) with severe consequences such as increased species extinction risk (Thomas et al. 2004) and sea level rise (Houghton et al. 2001). In the United States, buildings are the largest single contributor to carbon dioxide emissions, due to their vast use of energy which accounts for $40 \%$ of all primary energy use (EIA 2012). The carbon dioxide emissions from residences alone amounts to 1,184 million metric tons $\mathrm{CO}_{2}$ eq., or $22 \%$ of all U.S. carbon emissions (EPA 2012).

The energy demand of buildings, in particular residential buildings, has been found to be significantly affected by occupant behavior (Bahaj, Myers, and James 2007; Emery and Kippenhan 2006; Santin, Itard, and Visscher 2009; Yu et al. 2011). In residences occupants have a high level of control over their energy use through setting heating and cool points (or not using air conditioners or heaters), appliance use frequency, lighting use, etc. Variations in behavior practices have been found to create differences in energy consumption in identical buildings by more than a factor of two (Gill et al. 2011). Changing household behaviors has substantial potential to reduce carbon emissions (Dietz et al. 2009; Gardner and Stern 2008). Accordingly, numerous studies have taken place since the 1970s attempting to promote energy conservation in the home (Stern 2011; Wilson and Dowlatabadi 2007). Interventions have used many techniques to attempt to change behavior including: using prompts, providing information (attempting to change attitudes and providing procedural knowledge), making commitments, providing feedback, social 


\section{Anderson and Lee}

modeling, goal-setting, using norms, and attempts to manipulate intrinsic and extrinsic motivations (De Young 1993; Osbaldiston and Schott 2012). These interventions have shown various levels of success and can often bring about short term reductions in energy use (long term effects, however, have rarely been evaluated).

Recently, with advances in technology (e.g., home energy management systems), providing occupants with feedback as an intervention to reduce energy consumption use has been receiving increased attention. In particular, how comparative feedback and descriptive social norms can promote energy conservation has been investigated (Peschiera, Taylor, and Siegel 2010; Schultz et al. 2007). Due to the expense in applying interventions, researchers have begun using computer simulations to analyze potential outcomes from implementing comparative feedback interventions (Anderson, Lee, and Menassa 2013; Chen, Taylor, and Wei 2012). In these studies the authors developed agent-based models (ABM) that simulate installing an energy use feedback system into a building (e.g. installing home energy monitoring systems) where occupants can view the energy use of friends in their social network which in turn influences their energy use behavior. From these studies we have learned that the social network structure in the building can play an important role in intervention outcomes, but these studies have only evaluated social network effects using static social networks. Once relationships were made between agents in the models they never changed nor did people enter or leave the network. In Chen, Taylor, and Wei (2012) interventions were analyzed in the short term and in Anderson, Lee and Menassa (2013) the timeframe was not specified, so this assumption is plausible. However, to be able to consider the longer term implication of utilizing comparative peer feedback and group norm feedback interventions, this critical assumption is no longer sufficient.

Therefore, the objective of this study is to better understand how peer feedback interventions are affected as social networks evolve over time. More specifically we aim to answer two questions: 1) How do intervention outcomes, change in energy use, differ in dynamic as opposed to static social networks, and 2) do different patterns of energy use within the social network emerge as a result of becoming dynamic. This paper proceeds by describing the model that was created to simulate the spread of social norms in social networks along with its theoretical foundations and assumptions. Next we present the results and finally we conclude with a discussion of the results.

\section{METHODS}

In this section we detail our model using the ODD (Overview, Design concepts, Details) protocol for describing agent-based models (Grimm et al. 2006; Grimm et al. 2010) to improve the clarity, completeness and reproducibility of our model. We programmed the model in Java using Repast J v 3.0 (Repast 2013).

\subsection{Purpose}

This model has been developed to enhance our understanding of how normative behavior interventions, here peer group norm marketing, are affected by the dynamic characteristics of social networks.

\subsection{Entities, State Variables, and Scales}

The agents in this model are the building occupants. Occupants have multiple attributes. Each occupant has a particular energy practices which are summed up by their power rating, or energy use behavior (EUB). Agents also have relationships with other agents in the model through their social network. All relationships between occupants are reciprocal. Additionally, agents are assigned a chance to check their feedback on a given day and a value which determines how susceptible they are to the influence of their social group. Each time step in the simulation represents one day. 


\section{Anderson and Lee}

\subsection{Process Overview and Scheduling}

When the model is initialized it first creates all occupants that will be present during the first time step and sets their EUB and daily chance to check their feedback. Following this, the model creates the social network and assigns occupant relationships. Once the social network has been created the susceptibility of occupants to social influence is calculated based on their position in the network, this will be detailed in the submodel section.

Next the model begins to progress forward in time. During each time step, if the model is dynamic, every occupant has a chance to move out of the building, and thus the building's social network. If the model is not dynamic, this does not apply. If an occupant decides it is time to move and leaves the building (social network) all relationships to this occupant are severed and a new occupant immediately takes their place (the occupants are replaced immediately to make net energy use between models directly comparable). The new occupant is initialized with an EUB value and creates new friendships with existing occupants, how this works will be detailed later. The removal and addition of a new occupant requires that the susceptibility to peer influence of each occupant is recalculated. Additionally during each time step, every occupant has a chance to either randomly change their EUB in either direction or change their EUB by comparing with their peer group and conforming. After all occupants have had the opportunity to change their EUB the model updates synchronously and data is collected about energy used during that time step. The simulation run terminates after two years of simulated time.

\subsection{Design Concepts}

This model is built on the well-documented premise that people tend to conform to group norms (Schultz et al. 2007) and exhibit social learning. In the model occupants are provided feedback of peers in their social group and adjust their behavior to match that of their peers. An important aspect of occupant behavior in the model is its relative stability when not observing feedback, as people often develop automated responses to stimuli in their behavior setting, or habits, which are persistent. However, the model does account for 'unexplained' behavior changes by incorporating in stochasticity in behavior change. Allowing for factors beyond feedback to change the occupants' EUB is required to make agent behavior more realistic. It is well known that how humans determine to make decisions regarding behavior is extremely complex and subject to numerous determinants. Being able to account for fifty percent of the variance in behavior intention, not actual behavior, in psychological studies is considered very respectable.

The social network is constructed as a small-world network, which in effect consist of many tightly connected groups of individuals, i.e. clichés, all of which are loosely connected together (Watts and Strogatz 1998). To maintain a similar network structure when adding new occupants to the existing dynamic network, occupants are first randomly assigned to create a connection with one occupant in the network. For their subsequent relationships they are given a strong preference to create relationships with the friends of their existing friends. However, the new occupants, much the same as the original occupants, have a small chance to make a random connection to any other occupant in the network $(\mathrm{p}=0.1)$. This allows the social network to change overtime while maintaining a similar structure. Stochasticity is assumed here in making connections to peers, along with determining whether to move out of the building, whether to check feedback, and whether to change EUB due to other causes.

\subsection{Initialization}

Both the dynamic and static social networks are initialized with 49 occupants which simulates a small multi-unit apartment complex of renters. An occupant's EUB is generated from a log-normal distribution with a mean of 168 watts and standard deviation of 123 watts (Chen, Taylor, and Hei 2012). For both the dynamic and static networks, a small world network is generated using a probability of 0.1 to create random friendship connections. The network is generated using a regular ring lattice and each occupant generates two friendships. In the end each occupant averages four friendships since all relationships are recip- 
rocal in nature (see Anderson, Lee, and Menassa (2013) for more detail). The chance for random changes in behavior is set at ten percent and the chance occupants check their feedback is uniformly distributed between five and fifty percent since the frequency of checking feedback has been found to be variable (Jain, Taylor, and Peschiera 2012). Lastly, everyday occupants have a $0.0877 \%$ chance to move out (32\% annual turnover) (Ihrke, Faber, and Koerber 2011).

\subsection{Submodel}

\subsubsection{Social Influence Calculations}

When occupants check the feedback system, they update their EUB to conform to the mean of their peer group. At the same time occupants are slightly limited by their initial EUB. The method of calculating peer influence is based on the social network influence work of Friedkin (2001). We borrow his equations for calculating the spread of social influence and slightly simplify them by making each peer of the occupant being evaluated have equal influence on the occupant. The amount an occupant changes their EUB if they check their feedback system is determined by

$$
E U B_{i, t+1}=\left(1-a_{i, t}\right) E U B_{i, 1}+\frac{a_{i, t}}{F_{i, t}} \sum_{j}^{F_{i, t}} E U B_{j, t},
$$

where $E U B_{i, t+1}$ is the EUB of occupant $i$ at time $t+1, a_{i, t}$ is how susceptible occupant $i$ is to social influence at time $t, F_{i, t}$ is the number of friends occupant $i$ has at time $t$, and $E U B_{j, t}$ is the EUB of the $j$ - $t h$ friend of occupant $i$ at time $t$. Susceptibility of the agents is calculated each time an occupant either enters or leaves the network using the formula

$$
a_{i, t}=\left[1-\frac{1}{1+e^{-\left(F_{i, t}-2 F_{t}\right)}}\right]^{1 / 2}
$$

where $F$ is the mean number of relationships per occupant during the current time step. These formula hold except on the occasion when an occupant loses all of their relationships and gets completely separated from the rest of the social network. In this case, since the occupant is no longer subject to any social influence they now only change their EUB by the random change mechanism until they either leave the network or become reattached to others in the network.

\subsection{Experiment}

To evaluate the impact a dynamic social network structure has on comparative feedback interventions outcomes we ran one thousand simulations for both dynamic and static social networks using the same initialization values. Data is recorded throughout the simulation run at every time step. Three dependent variables are being collected for statistical analysis: total energy used over the course of the two year simulation period, change in mean EUB at the two year mark relative to initialization, and the mean standard deviation (SD) in EUB during the simulation runs. Monitoring total energy use permits us to see differences in the net outcome of installing feedback systems. Tracking the change in EUB from initiation to conclusion acts as a snapshot and provides insight in to how variable EUB is at a given time during the course of the intervention. The standard deviation of EUB helps identify what sort of dynamics are taking place within the model, i.e., do occupants tend to converge to a common norm or do other patterns of EUB emerge.

\section{RESULTS}

Two thousand simulation runs were conducted in total, one thousand simulating how implementing a comparative feedback system could be expected to function in a static social network and one thousand in 


\section{Anderson and Lee}

a dynamic network. The Kruskal-Wallis test was used for statistical analysis since the modeling assumptions for ANOVA could not be satisfied.

Total energy used over the course of two years for dynamic and static social network structures did not significantly differ $(H=0.0669, n . s ., d f=1)$. On the other hand, network dynamicity had a significant effect on the change in mean EUB from model initialization to the two year mark $(H=10.5113, p=$ $0.001186, d f=1$ ) (Figure 1). The difference in median values between the two configurations is relatively minor when considering that the model initialized occupants from a lognormal distribution with a mean EUB value of 168 watts $\left(M_{\text {Static }}=-1.495\right.$ watts, $M_{\text {Dynamic }}=-4.460$ watts $)$. However, the range of outcomes for mean EUB change at the two year mark for dynamic social networks is substantially larger, approximately twice as large. This pattern was also observed while watching single simulations runs. Fluctuations in mean EUB over the two years tend to be much less dramatic in the static social network (Figure 2).

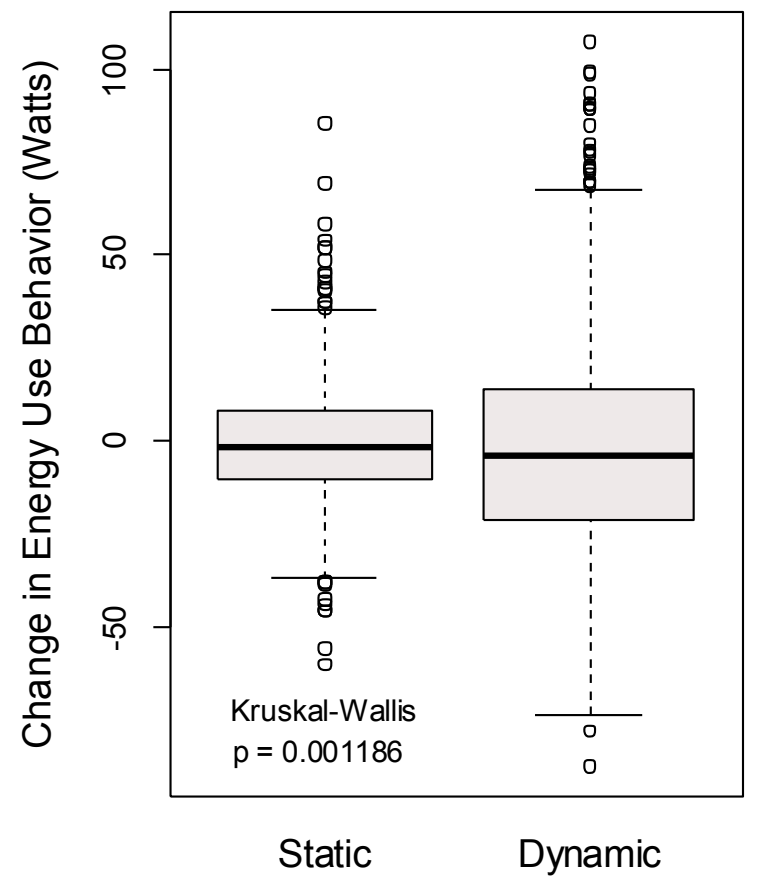

Social Network

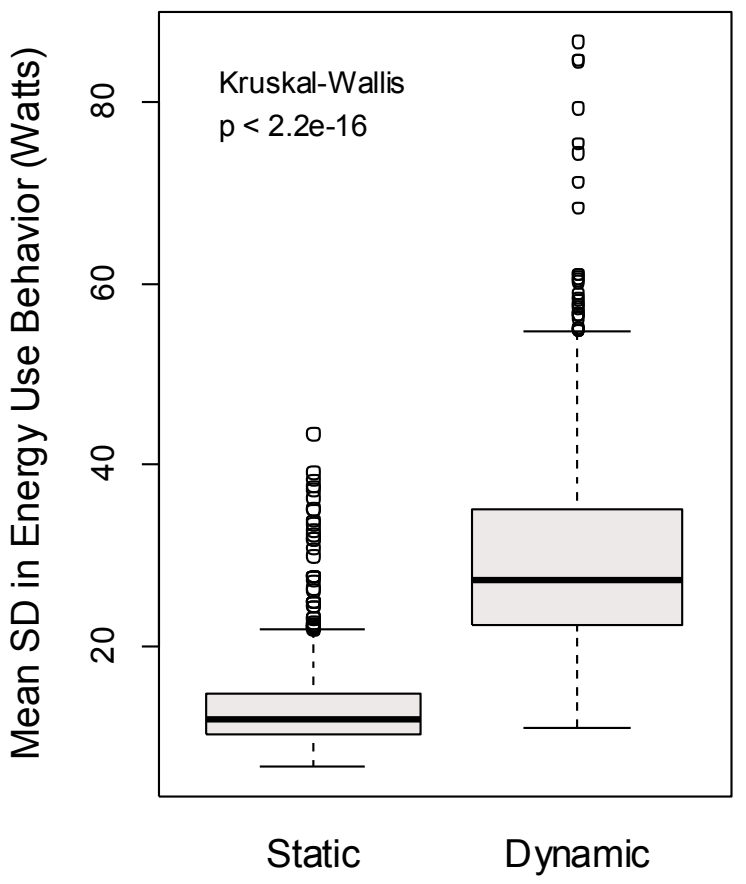

Social Network

Figure 1: Left - the relationship between social network structure dynamicity and the change in mean EUB from initialization to the two year mark is presented. Network dynamicity has a significant effect on change in mean EUB over the course of two years $(H=10.5113, p=0.001186, d f=1)$; however, differences in median values are not particularly large $\left(M_{\text {Static }}=-1.495\right.$ watts, $M_{\text {Dynamic }}=-4.460$ watts $)$. Right mean standard deviations in EUB in single runs by social network configuration are displayed. Social network dynamicity was found to have a significant effect on simulation run mean standard deviations in EUB $(H=1276.816, p<2.2 \mathrm{e}-16, d f=1)$. Sizeable differences in median values $\left(M_{\text {Static }}=12.115\right.$ watts, $M_{\text {Dynamic }}=27.300$ watts) of mean standard deviation in EUB suggests that dynamic networks have much higher levels of grouping behavior relative to static networks. Please note the difference in scales for the two boxplots.

The last measured variable is the mean SD of EUB throughout the two year period. Social network dynamicity was found to have a significant effect on simulation run mean SDs in EUB $(H=1276.816, p$ 


\section{Anderson and Lee}

$<2.2 \mathrm{e}-16, d f=1)$. Sizeable differences exist here in the median values $\left(M_{\text {Static }}=12.115\right.$ watts, $M_{\text {Dynamic }}=$ 27.300 watts) of mean SD in EUB. The range of observations once again is substantially wider for the dynamic social network relative to the static. Excluding outliers, the range of the mean SD of EUB for the dynamic social network simulations is roughly three times larger.
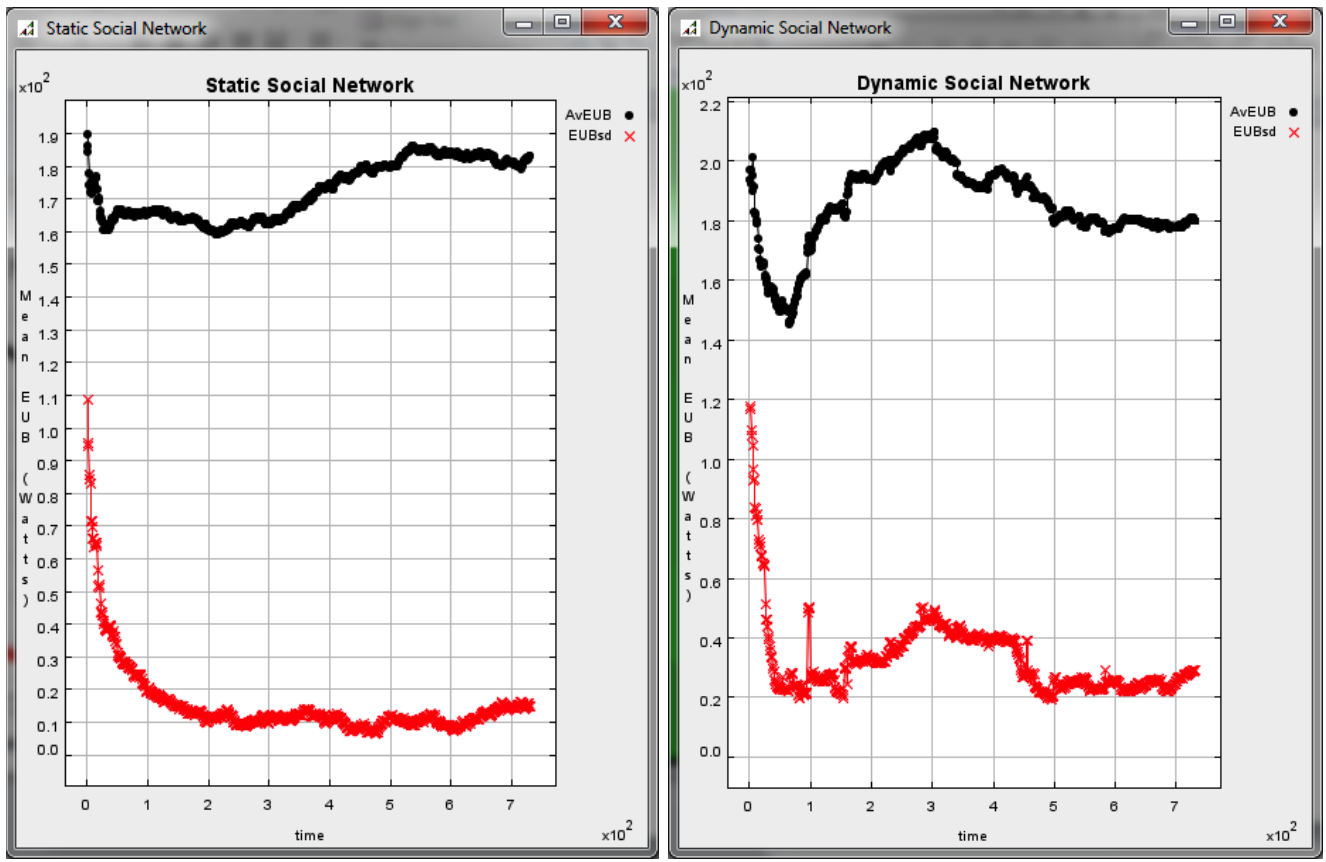

Figure 2: These imagines are of single run data for a static (left) and a dynamic social network (right) over the course of two years. The x-axis is time and the y-axis is the mean energy use behavior of the occupants (watts). Both networks are initialized using the same random seed, and the dynamic network exhibits much more volatile changes in behavior although both end around the same value. The black dots are the mean energy use behavior values of the occupants at each time step. The red x's are the corresponding standard deviation at that time. Please note the difference in scale for the y-axis.

\section{DISCUSSION AND CONCLUSIONS}

Results and observations from the experiment provide us with some insight into how behavior interventions targeting occupant energy consumption can be affected by social network dynamicity. In the static social network occupant behavior trends toward convergence as occupants alter their behavior to become more like their peers. This results in less dramatic shifts in mean EUB in the network. Dynamic social networks on the other hand exhibit substantially more volatile changes in mean EUB. Here as occupants enter and exit the social network, convergence of behavior network wide is largely prohibited. With the dynamic social networks, pockets, or groups, of energy users of various levels emerge. This is the same as in static network, but due to constant turnover (32\% annually) and changes in network structure these groups do fully spread their influence to other groups at a rate sufficient for system wide convergence.

Static and dynamic social networks showed no change in system level energy consumption in the experiments, which could be expected considering how occupants alter their EUB. Logically, if people are only to conform to social norms and have no other change in motives related to the behavior (e.g. sense of moral obligation), while individual behavior may improve or worsen as individuals alter their behavior to conform to the norm, the system level behavior should remain relatively constant all things being equal. This does not mean, however, that the simulation results do not have any important implications for modeling and performing occupant interventions. The results imply that for normative interventions dynamic 
social networks considerably increase uncertainty in intervention outcome over static social networks. Naturally, given that real world social networks are rarely if ever static, but rather are dynamic this suggests that current models simulating occupant interventions are possibly underestimating the range of potential outcomes. This is a critical because when proposing new interventions to facility or property managers, high levels of uncertainty in intervention outcome success, or failure, can provide a significant barrier to implementation.

\section{ACKNOWLEDGMENTS}

We would like to thank the University of Michigan Complex Systems Computer Laboratory for allowing us to use their computing resources.

\section{REFERENCES}

Abrahamse, W., L. Steg, C. Vlek, and T. Rothengatter. 2005. "A Review of Intervention Studies Aimed at Household Energy Conservation." Journal of Environmental Psychology 25(3):273-291.

Anderson, K., Lee, S., and Menassa, C. 2013. "Impact of Social Network Type and Structure on Modeling Normative Energy Use Behavior Interventions." Journal of Computing in Civil Engineering in press.

Bahaj, A.S., L. Myers, and P.A.B. James. 2007. "Urban Energy Generation: Influence of Micro-wind Turbine Output on Electricity Consumption in Buildings." Energy and Buildings 39(2): 154-165.

Chen, J., J. E. Taylor, and H-H. Wei. 2012. "Modeling Building Occupant Network Energy Consumption Decision-making: The Interplay Between Network Structure and Conservation." Energy and Buildings 47:515-524.

De Young, R. 1993. "Changing Behavior and Making It Stick The Conceptualization and Management of Conservation Behavior." Environment and Behavior 25:485-505.

Dietz, T., G. T. Gardner, J. Gilligan, P. C. Stern, and M. P. Vandenbergh. 2009. "Household Actions Can Provide a Behavioral Wedge to Rapidly Reduce US Carbon Emissions." Proceedings of the National Academy of Sciences of the United States of America 106(44):18452-6.

Emery, A.F., and C.J. Kippenhan. 2006. "A Long Term Study of Residential Home Heating Consumption and the Effect of Occupant Behavior on Homes in the Pacific Northwest Constructed According to Improved Thermal Standards." Energy 31(5):677-693.

Friedkin, N. E. 2001. "Norm Formation in Social Influence Networks." Social Networks 23 (3): 167-189.

Gardner, G. T., and P. C. Stern. 2008. "The Short List: The Most Effective Actions US Households Can Take to Curb Climate Change." Environment: Science and Policy for Sustainable Development 50(5):12-25.

Gill, Z. M., M. J. Tierney, I. M. Pegg, and N. Allan. 2010. "Low-energy Dwellings: The Contribution of Behaviours to Actual Performance." Building Research \& Information 38(5):491-508.

Grimm, V., U. Berger, F. Bastiansen, S. Eliassen, V. Ginot, J. Giske, J. Goss-Custard, et al. 2006. "A Standard Protocol for Describing Individual-based and Agent-based Models." Ecological Modelling 198(1-2):115-126.

Grimm, V., U. Berger, D. L. DeAngelis, J. G. Polhill, J. Giske, and S. F. Railsback. 2010. “The ODD Protocol: A Review and First Update." Ecological Modelling 221(23):2760-2768.

Houghton, J. T., Y. D. J. G. Ding, D. J. Griggs, M. Noguer, P. J. van der Linden, X. Dai, K. Maskell, and C. A. Johnson. 2001. Climate change 2001: the scientific basis. Vol. 881. Cambridge: Cambridge University Press.

Ihrke, D. K., C. S. Faber, and W. K. Koerber. 2011. “Geographical mobility: 2008 to 2009.” P20-565, US Department of Commerce, Economics and Statistics Administration, US Census Bureau.

Jain, R. K., J. E. Taylor, and G. Peschiera. 2012. "Assessing Eco-feedback Interface Usage and Design to Drive Energy Efficiency in Buildings." Energy and Buildings 48:8-17. 


\section{Anderson and Lee}

Osbaldiston, R., and J. P. Schott. 2012. "Environmental Sustainability and Behavioral Science: MetaAnalysis of Proenvironmental Behavior Experiments." Environment and Behavior 44(2):257299.

Peschiera, G., J. E. Taylor, and J. A. Siegel. 2010. "Response-relapse Patterns of Building Occupant Electricity Consumption Following Exposure to Personal, Contextualized and Occupant Peer Network Utilization Data." Energy and Buildings 42(8):1329-1336.

Repast. 2013. "Recursive Porus Agent Simulation Toolkit." Last retrieved May 1, 2013. http://repast.sourceforge.net/repast_3/.

Santin, O.G., L. Itard, and H. Visscher. 2009. "The Effect of Occupancy and Building Characteristics on Energy Use for Space and Water Heating in Dutch Residential Stock." Energy and Buildings 41(11):1223-1232.

Schultz, P.W., J. M. Nolan, R. B. Cialdini, N. J. Goldstein, and V. Griskevicius. 2007. "The Constructive, Destructive, and Reconstructive Power of Social Norms." Psychological Science 18(5):429-34.

Stern, P. C. 2011. "Contributions of Psychology to Limiting Climate Change." The American Psychologist 66(4):303-14.

Thomas, C. D., A. Cameron, R. E. Green, M. Bakkenes, L. J. Beaumont, Y. C. Collingham, B. F. N. Erasmus, et al. 2004. "Extinction Risk from Climate Change." Nature 427 (6970):145-148.

U.S. Energy Information Administration (EIA). 2012. "Annual Energy Review 2011: September 2012." DOE/EIA-0384(2011), Washington, DC.

U.S. Environmental Protection Agency (EPA). 2012. "Inventory of the U.S. Greenhouse Gas Emissions and Sinks: 1990-2010.” EPA 430-R-12-001, Washington, DC.

Watts, D. J., and S. H. Strogatz. 1998. "Collective dynamics of "small-world' networks." Nature 393(6684):440-2.

Wilson, C., and H. Dowlatabadi. 2007. "Models of Decision Making and Residential Energy Use." Annual Review of Environment and Resources 32(1):169-203.

Yu, Z., B. C. M. Fung, F. Haghighat, H. Yoshino, and E. Morofsky. 2011. "A Systematic Procedure to Study the Influence of Occupant Behavior on Building Energy Consumption." Energy and Buildings 43(6):1409-1417.

\section{AUTHOR BIOGRAPHIES}

KYLE ANDERSON is a Ph.D. student in the Civil and Environmental Engineering Department at the University of Michigan. He is interested in combining green building and behavioral approaches to reduce energy consumption in the built environment. He is particularly interested in investigating how we can promote generalizable and durable changes in occupant behavior through novel behavioral interventions. His email address is kyleand@umich.edu.

SANGHYUN LEE is an Assistant Professor at the University of Michigan. Before joining the faculty there, Dr. Lee worked as an Assistant Professor at the University of Alberta, Canada, from 2007 to 2010. He earned a Ph.D. in Construction Management and Information Technology from MIT, and worked at CRA International as a consultant and participated in numerous international construction projects while teaching at MIT (2006-2007). Dr. Lee's main research interest is the understanding and management of construction dynamics through the design and development of mechanisms, models, and systems that enhance proactive management of mega construction projects. His e-mail is shdpm@umich.edu. 